\title{
Highly Complex Magnetic Microstructures in Hierarchically Phase Separated AlCo(Cr)FeNi High- entropy Alloys
}

\section{Qianqian Lan ( $\sim$ q.lan@fz-juelich.de )}

Ernst Ruska-Centre for Microscopy and Spectroscopy with Electrons and Peter Gr" unberg Institute, Forschungszentrum J"ulich, $52425 \mathrm{~J}$ "ulich, Germany

\section{András Kovács}

Ernst Ruska-Centre for Microscopy and Spectroscopy with Electrons and Peter Grünberg Institute

\section{Jan Caron}

Ernst Ruska-Centre for Microscopy and Spectroscopy with Electrons and Peter Grünberg Institute

\section{Hongchu Du}

Forschungszentrum Jülich GmbH https://orcid.org/0000-0002-4661-4644

\section{Dongsheng Song}

Forschungszentrum Jülich

\section{Vishal Soni}

University of North Texas

\section{Sriswaroop Dasari}

University of North Texas https://orcid.org/0000-0001-5992-0299

\section{Varun Chaudhary}

Nanyang Technological University https://orcid.org/0000-0002-9244-1975

\section{Raju Ramanujan}

School of Materials Science and Engineering, Nanyang Technological University

\section{Rajarshi Banerjee}

University of North Texas

\section{Rafal Dunin-Borkowski}

Forschungszentrum Jülich https://orcid.org/0000-0001-8082-0647

\section{Article}

Keywords: hierarchical microstructures, high-entropy alloys (HEAs), coercivity

Posted Date: September 23rd, 2020

DOI: https://doi.org/10.21203/rs.3.rs-63802/v1 
License: (c) (i) This work is licensed under a Creative Commons Attribution 4.0 International License. Read Full License 


\section{Highly complex magnetic microstructures in hierarchi- cally phase separated $\mathrm{AICo}(\mathrm{Cr}) \mathrm{FeNi}$ high-entropy alloys}

Qianqian Lan ${ }^{1,2, *}$, András Kovács ${ }^{1}$, Jan Caron $^{1}$, Hongchu Du ${ }^{1,3}$, Dongsheng Song ${ }^{1}$, Soni Vishal ${ }^{4}$, Sriswaroop Dasari ${ }^{4}$, Varun Chaudhary ${ }^{5}$, Raju V. Ramanujan ${ }^{5}$, Rajarshi Banerjee ${ }^{4,5}$ \& Rafal E. Dunin-Borkowski ${ }^{1}$

${ }^{1}$ Ernst Ruska-Centre for Microscopy and Spectroscopy with Electrons and Peter Grünberg Institute, Forschungszentrum Jülich, 52425 Jülich, Germany

${ }^{2}$ School of Materials Science and Engineering, Tsinghua University, Beijing, 100086, China

${ }^{3}$ Central Facility for Electron Microscopy, RWTH Aachen University, 52074 Aachen, Germany

${ }^{4}$ Department of Materials Science and Engineering, University of North Texas, Denton, TX 76201, USA

${ }^{5}$ School of Materials Science and Engineering, Nanyang Technological University, Singapore 639798, Singapore

The hierarchical microstructures of high-entropy alloys (HEAs) can result in highly complex magnetic textures and properties. Here, we use high spatial resolution correlative magnetic, structural and chemical imaging to investigate magnetic textures in phase separated $\operatorname{AlCo}_{x} \mathrm{Cr}_{1-x} \mathrm{FeNi}(x=0.5$ and 1) HEAs. The AlCoFeNi HEA, which contains nm-sized A2 precipitates in a B2 matrix, supports large magnetic domains with small-angle magnetization variations. In contrast, the $\mathrm{AlCo}(\mathrm{Cr}) \mathrm{FeNi} \mathrm{HEA}$, which undergoes hierarchical phase separation, contains an unexpected distribution of magnetic vortices within individual A2 
precipitates in a weakly ferromagnetic B2 host, in addition to weakly ferromagnetic or nonmagnetic B2 precipitates in large magnetic domains of the A2 phase, as well as Fe-Co-rich inter-phase A2 regions that have strong magnetization. The coercivity is attributed to a complicated magnetization reversal process, which includes the successive reversal of the magnetic vortices. These results provide important insight for the rational design of HEAs with unique and tailored magnetic properties.

There is increasing demand for low cost energy-efficient rare-earth-free magnetic materials with superior magnetic and mechanical properties for applications such as wind power generators and high performance electric motors ${ }^{1}$. Al-Ni-Co (Alnico) permanent magnets, which are based on the Al-Ni-Co-Fe system, have hard magnetic properties (high coercivity $H_{\mathrm{C}}$ and energy product), with $H_{\mathrm{C}}$ values ranging from 48 to $202 \mathrm{mT}$, as well as a wide working temperature range up to $550{ }^{\circ} \mathrm{C}^{2}$. Their hard magnetic properties result from the shape anisotropy of a periodic Fe-Co hard magnetic phase, which is embedded in a non-magnetic Ni-Al-rich matrix ${ }^{2-4}$. The processing of such phase-separated magnets involves spinodal decomposition during high temperature heat treatment.

The use of near-equiatomic proportions of elements such as $\mathrm{Al}, \mathrm{Ni}, \mathrm{Co}, \mathrm{Fe}$ and $\mathrm{Cr}$ is used to form so-called high-entropy alloys (HEAs) ${ }^{5-7}$, in which high configurational entropy of mixing can promote solution formation. HEAs have attracted significant attention in the last decade because their rich composition and phase space provides opportunities for discovering alloys that have new mechanical and functional properties ${ }^{6-17}$. Previous studies have reported that $\mathrm{AlCo}(\mathrm{Cr}) \mathrm{FeNi}$ 
ferromagnetic HEAs have good mechanical properties and low coercivities of below $10 \mathrm{mT}$ when the constituent elements, processing and thermal history are controlled carefully ${ }^{18-21}$. However, the local origin of the dramatic difference between the magnetic properties of Alnico magnets and $\mathrm{AlCo}(\mathrm{Cr}) \mathrm{FeNi} \mathrm{HEAs}$ is almost unexplored.

The development of a full experimental understanding of the relationship between the local microstructure and magnetic texture of $\mathrm{AlCo}(\mathrm{Cr}) \mathrm{FeNi} \mathrm{HEAs}$ is crucial for controlling their mechanical and magnetic properties. The magnetic characteristics of HEAs are sensitive to the compositions and morphologies of the constituent phases over various length scales ${ }^{7,15,22,23}$. For example, the addition of paramagnetic or antiferromagnetic elements can induce phase segregation and decrease saturation magnetization $M_{\mathrm{S}}{ }^{15}$, the addition of $25 \% \mathrm{Cr}$ to FeCoNi can make the resulting FeCoNiCr alloy paramagnetic ${ }^{24}$, while the introduction of Mn to FeCoNiCrMn can eliminate the energy difference between face-centered-cubic and hexagonal-close-packed structures, resulting in magnetic frustration ${ }^{25}$. The magnetic properties of such highly heterogeneous alloys, in which each phase displays chemical and topological disorder, cannot be described as a compositionweighted average of the magnetic properties of the constituent phases ${ }^{22,23}$. An in-depth understanding of structure-magnetism correlations in complex concentrated multi-component systems has also been hindered by a lack of appropriate characterization methods.

Here, we use atom probe tomography (APT) and transmission electron microscopy (TEM) methods, including the Fresnel mode of Lorentz TEM and off-axis electron holography (EH), to investigate the influence of complex hierarchical phase separation of the A2 phase (disordered 
body-centered cubic) and the $\mathrm{CsCl}$-structured $\mathrm{B} 2$ phase (ordered body-centered cubic) on local variations in the magnetic microstructure of $\mathrm{AlCo}(\mathrm{Cr}) \mathrm{FeNi} \mathrm{HEAs}$. We study the local magnetic characteristics of the individual phases, including their saturation magnetic inductions and coercivities. Our results provide direct experimental measurements of correlations between microstructure and magnetic properties in HEAs with nanometer spatial resolution. This information is essential for understanding complex magnetism in multi-phase $\mathrm{AlCo}(\mathrm{Cr}) \mathrm{FeNi}$ alloys, as well as for the design of new HEAs with unique tailored magnetic properties.

\section{Results}

Magnetic properties of heat-treated AICoFeNi and AICo(Cr)FeNi bulk HEAs. Figure 1 shows magnetization $v s$ magnetic field $(M-H)$ hysteresis loops measured at $300 \mathrm{~K}$ for the heat-treated $\mathrm{Al}-$ $\mathrm{CoFeNi}$ and $\mathrm{AlCo}(\mathrm{Cr}) \mathrm{FeNi}$ alloys. The loops suggest soft ferromagnetic properties. The annealed AlCoFeNi HEA has a saturation magnetization $M_{S}$ of $99.8 \mathrm{emu} / \mathrm{g}$ and a coercivity $H_{c}$ of $1.45 \mathrm{mT}$. When half of the Co content is replaced by $\mathrm{Cr}$ in the annealed $\mathrm{AlCo}(\mathrm{Cr}) \mathrm{FeNi} \mathrm{HEA}, M_{S}$ decreases to $46.2 \mathrm{emu} / \mathrm{g}$, while $H_{c}$ increases to $9.56 \mathrm{mT}$. Both specimens are almost fully saturated magnetically at fields of $500 \mathrm{mT}$. It has been observed before in heat-treated $\mathrm{AlCo}(\mathrm{Cr}) \mathrm{FeNi}$ that $\mathrm{Cr}$ substitution and annealing induce complex hierarchical phase segregation ${ }^{20,21}$, which is thought to define the resulting magnetic properties. It is therefore important to understand the influence of each phase on the soft magnetic properties of AlCoFeNi and $\mathrm{AlCo}(\mathrm{Cr}) \mathrm{FeNi}$ HEAs.

Microstructural and magnetic properties of heat-treated AICoFeNi. The AlCoFeNi HEA stud- 
ied here has a polycrystalline microstructure with a grain size of above $100 \mu \mathrm{m}$. Figure $2 \mathrm{a}$ shows an High-angle annular dark-field (HAADF) scanning TEM (STEM) image of a grain boundary region between two adjacent $\mathrm{AlCoFeNi}$ grains. Grain 1 was aligned in the electron microscope to the closest crystallographic zone axis, at which an $\langle 001\rangle$ direction was parallel to the incident electron beam direction. In this projection, chemically-sensitive contrast reveals inhomogeneities. 3D APT studies of AlCoFeNi have previously revealed the presence of nm-sized Fe-Co-rich A2 precipitates in an Al-Ni-rich B2 matrix ${ }^{21}$. Figure $2 \mathrm{~b}$ shows $\mathrm{Al}$ and Fe elemental distributions masured using energy-dispersive X-ray spectroscopy (EDXS) in grain 1, confirming the presence of compositional variations. The average sizes of the Al-rich and Fe-rich regions are approximately $10 \mathrm{~nm}$. Figures $2 \mathrm{c}$ and $2 \mathrm{~d}$ show an atomic-resolution image and a corresponding elemental map of the Al-rich B2 phase and the Fe-rich A2 phase. Chemical ordering in the B2 phase is visible in the HAADF STEM image. The structures are almost coherent, with occasional dislocations at the interface due to the lattice misfit between them. The magnetic microstructure at remanence was initially studied using Fresnel defocus imaging in Lorentz mode. Figure 2e shows a representative overfocus Fresnel image recorded from grain 2. This region contains large magnetic domains, which are separated by magnetic domain walls that appear as black and white lines in the image. The ripple-like contrast variations in the domains originate from small-angle magnetization variations in the specimen. Figure $2 \mathrm{f}$ shows an approximate representation of the projected in-plane magnetic induction obtained from a pair of such defocused images using the transport-of-intensity equation $^{26,27}$. The magnetic domain configuration in the thin specimen was observed to rearrange upon applying a magnetic field as small as $5 \mathrm{mT}$ perpendicular to the specimen using the conven- 
tional electron microscope objective lens, suggesting isotropic soft magnetic behavior, in which the phase separated structure is not strong enough to pin the magnetic domain walls.

Microstructural properties of heat-treated AICo(Cr)FeNi. Figure 3a shows that the annealed $\mathrm{AlCo}(\mathrm{Cr}) \mathrm{FeNi} \mathrm{HEA}$ specimen has a strikingly different microstructure from that of the $\mathrm{AlCoFeNi}$ alloy, comprising an Al-Ni-Co-rich B2 phase, Fe-Cr-Co-rich A2 regions and their combinations. The different length scales of the A2 phases are referred to here as a) coarse A2 gen-1 (with an average size of 1-5 $\mu \mathrm{m}), \mathrm{b})$ medium-scale A2 gen-2 (50-150 nm) and c) fine-scale A2 gen-3 $(<10 \mathrm{~nm})$. Two characteristic regions are labeled R1 (A2 gen-2 + A2 gen-3 + B2 matrix) and R2 (A2 gen-1 + B2 matrix). An additional A2 phase (A2 shell) is observed between the B2 and A2 phases (see below), taking the form of a few-nm-thick shell around the A2 gen-1 and A2 gen-2 phases. Figure $3 b$ shows a schematic diagram of the constituent phases and regions in the $\mathrm{AlCo}(\mathrm{Cr}) \mathrm{FeNi}$ HEA specimen.

Figure 4 shows TEM and APT measurements of the shell A2 phase. Figure 4a shows an APT reconstruction of the elemental distribution in a needle-shaped specimen of region R1 in the $\mathrm{AlCo}(\mathrm{Cr}) \mathrm{FeNi} \mathrm{HEA}$, revealing the presence of small Fe-Cr-rich A2 gen-3 precipitates and large A2 gen-2 precipitates surrounded by Fe-Co-rich A2 shells in a B2 matrix. A detailed description of the composition of the interface between the B2 and A2 phases is presented in the Supplementary Information. Figures $4 \mathrm{~b}-4 \mathrm{~d}$ show an HAADF STEM image and corresponding Co and Fe EDXS elemental maps of the shell region. The enhancement in $\mathrm{Co}$ and $\mathrm{Fe}$ at the edges of the A2 gen-2 precipitate appears to be discontinuous, perhaps because of the geometry of the thin TEM 
specimen, from which part of the precipitate may have been removed by ion milling. Figure $4 \mathrm{e}$ shows an atomic-resolution HAADF STEM image of A2 gen-3 precipitates in the B2 matrix. Figures $4 \mathrm{f}$ and $4 \mathrm{~g}$ show atomic-resolution HAADF STEM images of the A2 structure of the shell, whose thickness is $2 \mathrm{~nm}$ around the A2 gen- 2 precipitates and $7.5 \mathrm{~nm}$ around the A2 gen-1 island. The measurements are consistent between the APT result and the EDXS images shown in Figs $4 \mathrm{~h}$ and $4 \mathrm{i}$. The $\mathrm{A} 2$ shell is rich in $\mathrm{Fe}$ and $\mathrm{Co}$, whereas little or no $\mathrm{Al}$ or $\mathrm{Cr}$ is present inside it. Atomic-resolution HAADF STEM images of the interface region are discussed in detail in the Supplementary Information.

\section{Magnetic microstructure of $\mathrm{AlCo}(\mathrm{Cr}) \mathrm{FeNi}(\mathrm{I})$ - A2 gen-2 precipitates in the B2 matrix (re-}

gion R1). Highly heterogeneous systems in which each region contains both chemical and topological disorder, such as heat-treated $\mathrm{AlCo}(\mathrm{Cr}) \mathrm{FeNi} \mathrm{HEAs}$, typically have complex magnetic properties in the constituent phases that cannot be understood from bulk magnetic measurements alone. We studied the local magnetic properties of the A2 and B2 phases in the R1 and R2 regions of the $\mathrm{AlCo}(\mathrm{Cr}) \mathrm{FeNi}$ specimen using Fresnel defocus imaging and off-axis EH. The latter technique provides a direct quantitative measurement of the phase shift of the electron wave that interacted with the specimen, from which the local magnetic state of the region of interest can be determined with nm spatial resolution ${ }^{28}$. The total electron optical phase shift contains electrostatic and magnetic contributions that need to be separated to measure the magnetic field distribution in the specimen. In the absence of electron-beam-induced charging, the electrostatic phase shift comprises primarily the mean inner potential (MIP) of the specimen, which depends on its composition, density and ionicity. 
Figure 5a shows the MIP contribution to the electron optical phase shift in region R1 measured using off-axis EH. In this image, the A2 gen-2 precipitates, which have close-to-spherical morphologies and diameters of between 50 and $120 \mathrm{~nm}$, appear brighter than the surrounding B2 matrix, as they have a higher mean atomic number per unit volume. Figure $5 \mathrm{~b}$ shows the corresponding magnetic contribution to the phase shift $\varphi_{M}$, which provides a measure of the in-plane component of the magnetic induction within and outside the specimen integrated in the electron beam direction ${ }^{29}$. Bright or dark contrast is visible within the boundaries of the A2 gen-2 precipitates, which are each surrounded by a thin Fe-Co-rich A2 shell. Figure 5c shows a magnetic induction map obtained by generating contour lines and colors from the magnetic contribution to the phase shift and its gradient, respectively. This image reveals that each A2 precipitate contains a magnetic vortex, with the magnetic field rotating either clockwise or counterclockwise. Similar 3D magnetic vortex states have been observed in sub-100-nm spherical Fe-Ni particles without strong magnetocrystalline anisotropy ${ }^{30,31}$. In region $\mathrm{R} 1$, the A2 precipitates are well separated by the B2 matrix, preventing dipolar interactions between individual crystals. The ratio of clockwise to counterclockwise magnetic vortices is approximately 1:1 at remanence after saturating the specimen using the microscope objective lens, suggesting energetically-independent magnetic states that are only weakly coupled to the surrounding phases. The vortices are not associated with significant measurable stray magnetic fields that could be measured at remanence using either bulk magnetometry or surface-sensitive magnetic characterization techniques.

A model-based iterative reconstruction algorithm ${ }^{32}$ was used to convert the measured magnetic phase images $\varphi_{M}$ into maps of projected in-plane magnetization $M_{x y}$, as shown in Fig. 5d for 
the two A2 gen-2 precipitates marked in Fig. 5b. The magnetization direction of the vortex core is parallel to the incident electron beam direction in the center of each precipitate and does not contribute to the projected in-plane magnetization map in this region. In general, each magnetic vortex core can point either up or down magnetically, irrespective of the vortex rotation direction

33. The magnetization direction of the vortex core cannot be detected from these images, as EH is sensitive to the component of the magnetic field that is perpendicular to the incident electron beam direction. An upper limit for the magnetic vortex core diameter was measured to be $\sim 8 \mathrm{~nm}$ by fitting a Gaussian function to the projected in-plane magnetization distribution. On the assumption that the specimen is magnetically active through its entire thickness, the magnitude of the projected in-plane magnetic induction (Fig. 5d) peaks at $0.5 \pm 0.1 \mathrm{~T}$ in the $\mathrm{A} 2$ gen-2 precipitates.

The Fe-Cr-Co-rich A2 gen-2 precipitates are covered by Fe-Co-rich A2 shells (Fig. 4). The measured magnetic signal is therefore a superposition of contributions from the two A2 phases. Non-uniform magnetization distributions in some of the A2 precipitates may result from their "incomplete" morphologies in an ion-milled TEM specimen. 3D tomographic reconstruction was used to clarify the shapes and distributions of the A2 gen-2 precipitates in the B2 matrix from a tilt series of ADF STEM images ${ }^{34}$. Figure 5e shows sections through a tomographic reconstruction of region R1 that contains approximately spherical A2 gen-2 precipitates (yellow) in a B2 matrix (blue). Some of the precipitates intersect the TEM specimen surface and are incomplete.

Magnetic switching of A2 gen-2 precipitates in the B2 matrix (region R1). The magnetic switching properties of A2 gen-2 precipitates in the B2 matrix (region R1) were studied by ap- 
plying magnetic fields perpendicular to the specimen plane. In situ magnetization reversal was performed by applying magnetic fields of up to $1.5 \mathrm{~T}$ using the conventional microscope objective lens. Figures $6 \mathrm{a}$ and $6 \mathrm{~b}$ show magnetic phase images of region $\mathrm{R} 1$ recorded after returning to remanence from opposite out-of-plane fields of $-500 \mathrm{mT}$ and $500 \mathrm{mT}$, respectively. The proportion of clockwise and counterclockwise magnetic vortices in the A2 gen-2 precipitates in region R1 was measured from the magnetic phase images. A comparison of Figs $6 \mathrm{a}$ and $6 \mathrm{~b}$ shows that the majority of the A2 gen-2 precipitates changed their magnetic field rotation direction. Within the field of view of approximately $1.4 \mu \mathrm{m} \times 1.4 \mu \mathrm{m}, 46$ counterclockwise and 34 clockwise vortices are visible in Fig. 6a, whereas 35 counterclockwise and 45 clockwise vortices are visible in Fig. 6 b. White squares in Fig. $6 \mathrm{~b}$ mark precipitates that retained the same sense of magnetic rotation as in the initial remanent state.

Figure $6 \mathrm{c}$ shows the magnitude of the local change in magnetic phase shift $\varphi_{m}$ recorded from representative A2 gen-2 precipitates of different size, plotted as a function of applied magnetic field from $05000 \mathrm{mT}$. The positive (or negative) sign of $\varphi_{m}$ is related to a counterclockwise (or clockwise) magnetic field rotation direction in the vortices. Two large A2 precipitates with diameters of 75 and $80 \mathrm{~nm}$ show a continuous decrease in magnetic phase shift close to zero as the applied magnetic field is increased to $500 \mathrm{mT}$, suggesting that the internal field in the precipitates becomes aligned parallel to the electron beam direction. As the applied magnetic field is decreased (Fig. 6c), the magnetic phase shift recovers, but with opposite sign, indicating that the magnetic vortex now has a rotation sense opposite to the original rotation direction. Different switching behavior was observed for a small A2 precipitate with a diameter of $55 \mathrm{~nm}$ (Fig. 6c). The initial 
rotation sense is counterclockwise at $0 \mathrm{mT}$, changes sign at $200 \mathrm{mT}$ and decreases gradually to zero as the applied magnetic field increases to $500 \mathrm{mT}$. A possible scenario is that the magnetic field direction of the vortex core was aligned antiparallel to the saturating magnetic field. At $200 \mathrm{mT}$, the vortex core switches to become aligned with the saturating field, which also changes the vortex rotation direction. As the applied magnetic field is increased further, this state becomes aligned with the applied field direction and the magnetic phase shift approaches zero. On decreasing the applied magnetic field, a magnetic vortex forms again at $400 \mathrm{mT}$ and remains stable as the applied magnetic field is reduced to zero.

The magnetic nature of the B2 matrix in region $\mathrm{R} 1$, which contains more than $60 \% \mathrm{Fe}, \mathrm{Co}$ and Ni according to APT and EDXS measurements (Fig. 4), is now discussed. Figure 6d shows a plot of the magnetic phase shift $\varphi_{m}$ in the B2 matrix in region R1 before and after magnetization reversal, revealing a region with a gradient in phase and an associated step $\Delta \varphi_{m} \sim 1.3 \mathrm{rad}$. The greatest intensity maxima and minima in the plot correspond to A2 gen-2 magnetic vortices that changed their rotation direction during switching. Changes in the sign of the magnetic phase shift $\varphi_{m}$ indicate that part of the Al-Ni-Co-rich B2 matrix is also magnetized in the plane of the specimen, is ferromagnetic and reverses in sign magnetically. Dipolar or magnetostatic interactions ${ }^{35}$ between the A2 precipitates are expected to be affected by the nature of the surrounding magnetic phases and inter-phase boundaries. It is noteworthy that the same magnetic contrast is observed in the B2 matrix, but with a sign change in the magnetically-switched region R1 (Fig. 6).

The in situ magnetic switching experiments reveal details about the magnetic properties of 
region $\mathrm{R} 1$ in annealed $\mathrm{AlCo}(\mathrm{Cr}) \mathrm{FeNi} \mathrm{HEAs}$ that contain A2-type precipitates in a $\mathrm{B} 2$ matrix. However, the lack of information about the core direction from off-axis EH experiments limits our understanding of the details of the process. The switching characteristics of the magnetic vortices depend on the sizes and shapes of the A2 gen-2 precipitates, the external magnetic field and coupling to the B2 matrix. Further analysis of this complex system requires comparisons of experimental measurements with atomistic spin dynamics or micromagnetic calculations that are beyond the scope of the present paper.

\section{Magnetic properties of $\mathrm{AlCo}(\mathrm{Cr}) \mathrm{FeNi}$ (II) - B2 precipitates in the A2 gen-1 matrix (region}

R2). The $\mathrm{AlCo}(\mathrm{Cr}) \mathrm{FeNi}$ alloy contains regions (R2) of micrometer-sized Fe-Cr-Co-rich A2 gen1 matrix with B2 precipitates. Microstructural and chemical studies show that an A2 shell is present between the B2 and A2 phases (Fig. 4). Figure 7a shows a Fresnel defocus image of an R2 island surrounded by R1 recorded at a defocus value of $-200 \mu \mathrm{m}$. The magnetic nature of the R2 phase is apparent from the presence of dark and bright bands of contrast. The image also shows the limitation of Fresnel defocus imaging, as the phase boundaries give rise to strong fringing fields at their edges, making it difficult to interpret the magnetic state. Figures $7 \mathrm{~b}, 7 \mathrm{c}$ show the MIP contribution to the phase and a corresponding magnetic induction map recorded using off-axis EH. In Fig. 7b, the A2 gen-1 and gen-2 (bright contrast) and B2 (dark contrast) phases can be distinguished, as they have different mean atomic numbers per unit volume. Selected B2 precipitates are marked by white frames in both images. The color-coded magnetic induction map shows that the $\mathrm{R} 2$ region contains large magnetic domains. The magnetic field lines are either disrupted or missing at the B2 precipitates, suggesting that they are weakly magnetic or non- 
magnetic. The effect of the smaller $(<50 \mathrm{~nm}) \mathrm{B} 2$ precipitates on the magnetic field is less clear, as it can be masked by the signal from the A2 gen-1 matrix in the $\sim 100$-nm-thick TEM specimen. Figure $7 \mathrm{~d}$ shows a line profile of the magnetic phase shift across region R2, which contains a single $\mathrm{B} 2$ precipitate at its center. The weakly magnetic or non-magnetic Al-Ni-Co-rich $\mathrm{B} 2$ precipitate has a lower contribution to the magnetic phase shift and appears as a dip. Close inspection of the phase profile in Fig. 7e reveals a change in slope at the position of the A2 shell, suggesting a difference in its magnetic properties from those of the A2 gen-1 matrix. The slope of the phase in the A2 shell and the A2 gen-1 matrix in region R2 are 0.075 and $0.06 \mathrm{rad} / \mathrm{nm}$, respectively, based on fitted linear functions. As the magnetic phase shift scales with the projected in-plane magnetic induction in the specimen, it can be inferred that the A2 shell has approximately $25 \%$ higher magnetization than the A2 gen-1 matrix in region $\mathrm{R} 2$. This difference is thought to result from the higher concentration of $\mathrm{Cr}$ in the $\mathrm{A} 2$ gen-1 matrix, which decreases the magnetization of the Cr-Fe-Co-rich A2 gen-1 matrix in the $\mathrm{R} 2$ region.

\section{Discussion}

Two important properties of $\mathrm{AlCo}(\mathrm{Cr}) \mathrm{FeNi}$ HEAs that are affected strongly by the presence of nonmagnetic $\mathrm{Al}$ and antiferromagnetic $\mathrm{Cr}$ are their saturation magnetization and coercivity. The reference AlCoFeNi HEA contains nm-sized Fe-Co-rich A2 precipitates in an Al-Ni-rich B2 matrix. The saturation magnetic induction of AlCoFeNi of $0.987 \mathrm{~T}(99.8 \mathrm{emu} / \mathrm{g})$ is associated primarily with ferromagnetic ordering of $\mathrm{Fe}, \mathrm{Co}$ and $\mathrm{Ni}$. The effect of $\mathrm{Cr}$ substitution and heat treatment of AlCoFeNi leads to decomposition of the alloy into an interesting duplex distribution of A2 and B2 
phases in regions R1 and R2. Bulk measurements of microstructure and magnetic properties are not able to resolve and provide an understanding of the separate contributions of each phase. The high spatial resolution analyses that are presented here reveal striking contributions of the details of the microstructure to the magnetic properties. Upon annealing the alloy to $600{ }^{\circ} \mathrm{C}$, interdiffusion of $\mathrm{Al}$ and $\mathrm{Cr}$ define the microstructure and magnetic properties of the specimen. $\mathrm{Cr}$ concentrates in the Fe-Cr-Co-rich A2 phase, which dominates the R2 islands and forms close-to-spherical magnetic precipitates in the $\mathrm{R} 1$ phase. A small amount of $\mathrm{Cr}$ can also be found in the Fe-Co-rich A2 shell. It is known from previous work that $\mathrm{Cr}$ aligns antiferromagnetically with $\mathrm{Fe}$ and $\mathrm{Co}^{36}$ and reduces the saturation magnetic moment. Bulk magnetometry measurements indicate that the addition of Cr to AlCoFeNi reduces the saturation magnetic induction from 0.987 to $0.46 \mathrm{~T}(46.2 \mathrm{emu} / \mathrm{g}){ }^{21}$, as a result of the presence of magnetic and non-magnetic phases in the specimen.

There are three primary contributions to the saturation magnetic induction: (i) $\mathrm{Fe}-\mathrm{Cr}$-Co-rich A2 gen-2 and $\mathrm{A} 2$ gen-3 precipitates in region $\mathrm{R} 1$ and the $\mathrm{A} 2$ gen-1 matrix in region $\mathrm{R} 2$; (ii) the $\mathrm{Fe}-$ Co-rich A2 shell between the A2 and B2 phases; (iii) the Al-Ni-rich B2 phase. The magnetic state of each phase is distinctly different. The first two contributions are strongly linked, as they form core-shell structures with thin A2 shells around A2 spheres or islands. Off-axis EH measurements reveal that the A2 shell in region R2 (Fig. 7) has a higher magnetic induction than the A2 core. A magnetic interaction is expected to be present between the two ferromagnetic A2 phases and to affect magnetization reversal. The A2 spheres in region $\mathrm{R} 1$ support 3D magnetic vortex states in the B2 matrix. Based on the magnetic phase shift measurement and on the result of model-based iterative reconstruction of projected in-plane magnetization, the saturation magnetic induction in 
the A2 spheres, which have a core-shell structure, is estimated to be approximately $0.5 \mathrm{~T}$ (Fig. 5). For a 2.6-nm-thick shell around a core with a radius of $40 \mathrm{~nm}$, the shell occupies almost $20 \%$ of the total volume. Therefore, the contribution of the thin Fe-Co-rich A2 shell to the total magnetization is significant. A magnetic contribution is also expected from the B2 phase, which contains more than $50 \%$ of $\mathrm{Fe}$, Ni and Co. Our magnetic phase images (Fig. 6) provide evidence for a magnetic signal in the B2 matrix.

In a multicomponent alloy, the coercivity $H_{c}$ is expected to be sensitive to impurities, deformation, grain size and phase decomposition ${ }^{37}$. The annealed AlCoFeNi HEA has a smaller value of $H_{c}$ than the $\mathrm{AlCo}(\mathrm{Cr}) \mathrm{FeNi} \mathrm{HEA}$. The magnetic microstructure in the AlCoFeNi HEA is characterized by large magnetic domains with small-angle magnetization variations. Magnetic domain walls then move easily in the presence of an applied magnetic field. In contrast, in the $\mathrm{AlCo}(\mathrm{Cr}) \mathrm{FeNi}$, which has a multi-scale hierarchical $\mathrm{B} 2+\mathrm{A} 2$ decomposed microstructure, phase boundaries between $\mathrm{R} 1$ and $\mathrm{R} 2$ regions can act as pinning sites for magnetic domain walls, thereby restricting their movement and resulting in an increase in coercivity $H_{c}$ compared to that in AlCoFeNi. Our magnetic switching study shows that, in region R1, magnetic vortices in A2 gen-2 precipitates can change their rotation direction in the presence of external magnetic fields of 100 to $500 \mathrm{mT}$. The smaller the diameter of the A2 gen-2 precipitate, the lower is the magnetic field that is needed to change the magnetic vortex rotation direction, suggesting that the coercivity of such an $\mathrm{AlCo}(\mathrm{Cr}) \mathrm{FeNi} \mathrm{HEA}$ can be tuned by changing the sizes and separations of the constituent $\mathrm{A} 2$ precipitates and islands. 
It is interesting to draw an analogy between phase-separated magnetic HEAs and Alnico alloys, in which a duplex nanoscale structure of two phases forms during thermal annealing in the presence of an external magnetic field, with anisotropic growth of a periodic Fe-Co hard magnetic phase in an Al-Ni-rich matrix resulting in shape anisotropy and enhanced coercivity. It is therefore of interest for future studies of magnetic HEAs to determine how the effect of field annealing and other external stimuli can be used to control magnetic anisotropy. In this way, the soft magnetic properties can be tuned in a similar way to that already successfully demonstrated for Alnico.

\section{Conclusions}

The magnetic microstructure of $\mathrm{AlCo}_{x} \mathrm{Cr}_{1-x} \mathrm{FeNi}(x=0.5$ and 1$)$ heat-treated HEAs has been investigated with unprecedented spatial resolution using off-axis EH and Lorentz TEM, in combination with 3D APT, STEM imaging and spectroscopy. In a simpler AlCoFeNi alloy, which contains nmsized A2 Fe-Co-rich precipitates in a B2 matrix, the magnetic structure is characterised by large magnetic domains and small-angle magnetization variations. In contrast, the substitution of Co by $\mathrm{Cr}$ in an $\mathrm{AlCo}(\mathrm{Cr}) \mathrm{FeNi}$ alloy results in the formation of two characteristic phases: (i) a ferromagnetic A2 phase in a weakly-magnetic B2 matrix and (ii) B2 precipitates in a magnetic A2 matrix. In the first phase, the A2 precipitates are approximately spherical and, surprisingly, contain individual magnetic vortices. In the second phase, the $\mathrm{B} 2$ precipitates disrupt otherwise-continuous magnetic domains in the A2 matrix. In addition, the presence of an Fe-Co-rich A2 shell between the B2 and A2 phases provides an additional contribution to the overall magnetization. The saturation magnetization of the $\mathrm{AlCo}(\mathrm{Cr}) \mathrm{FeNi} \mathrm{HEA}$ is dominated by the $\mathrm{Fe}-\mathrm{Cr}$-Co-rich $\mathrm{A} 2$ phases 
in both regions, as well as by the Fe-Co-rich $\mathrm{A} 2$ shells, whereas the $\mathrm{B} 2$ matrix phase provides a minor contribution. Its value is decreased by the substitution of $\mathrm{Co}$ by $\mathrm{Cr}$ as a result of the antiferromagnetic ordering nature of $\mathrm{Cr}$. The increased coercivity of the $\mathrm{AlCo}(\mathrm{Cr}) \mathrm{FeNi}$ HEA can be attributed to a complicated magnetization reversal process, which involves the reversal of magnetic vortices in a weakly-ferromagnetic matrix. Our results provide direct local information about the intricate complexity of magnetic remanent states and reversal processes in multicomponent HEAs that contain coexisting magnetic phases and hierarchical microstructures that span multiple length scales.

\section{Methods}

Specimen preparation. $\mathrm{AlCo}_{x} \mathrm{Cr}_{1-x} \mathrm{FeNi}(x=0.5$ and 1$)$ bulk specimens were prepared by arc melting $\mathrm{Al}, \mathrm{Co}, \mathrm{Cr}, \mathrm{Fe}$ and $\mathrm{Ni}$ pellets in an $\mathrm{Ar}$ atmosphere, followed by annealing at $600{ }^{\circ} \mathrm{C}$ for $15 \mathrm{~h}$ in an Ar atmosphere and quenching in water, as described elsewhere ${ }^{21}$. Bulk magnetometry measurements were performed in a vibrating sample magnetometer (VSM-Lakeshore 7404) using a maximum magnetic field of $1 \mathrm{~T}$. Specimens for TEM and APT were prepared using focused Ga ion beam milling in FEI Helios Nanolab 400s and FEI Nova Nanolab 20 dual beam systems following a standard lift-out method. Electron-transparent $(\sim 100 \mathrm{~nm})$ lamellae were attached to $\mathrm{Cu}$ Omniprobe support grids for TEM measurements.

Atom probe tomography. APT experiments were conducted in a LEAP 300X local electrode atom probe system (Cameca Instruments, Inc.). All atom probe experiments were conducted in electric field evaporation mode at a temperature of $60 \mathrm{~K}$ using an evaporation rate of $0.5 \%$ and 
a pulsing voltage of $20 \%$ of the steady-state applied voltage. Data analysis was performed using IVAS 3.6.2 software.

Transmission electron microscopy. HAADF STEM imaging, EDXS mapping and electron tomography were performed in an FEI Titan G2 80-200 electron microscope equipped with a high brightness field emission gun, a probe aberration corrector and an in-column Super-X EDXS system. HAADF STEM images were recorded on a Fischione detector using a beam convergence semi-angle of $24.7 \mathrm{mrad}$ and an inner detector semi-angle of $69 \mathrm{mrad}$.

Off-axis electron holography. The same specimens that were used for microstructural characterization were used to study magnetic texture using Lorentz microscopy and off-axis EH. Off-axis electron holograms were recorded in magnetic-field-free conditions (i.e., in Lorentz mode) in an image-aberration-corrected FEI Titan 80-300 electron microscope equipped with a high brightness field emission gun, an electron biprism, and a (Gatan K2 IS) direct electron counting detector ${ }^{40}$ camera using a typical exposure time of $6 \mathrm{~s}$. The biprism voltage was typically set to $100 \mathrm{~V}$, resulting in an overlap interference width of $2.1 \mu \mathrm{m}$ and a holographic interference fringe spacing of $2.76 \mathrm{~nm}$ with a contrast of $48 \%$ in vacuum. The objective lens of the microscope was used to apply out-of-plane magnetic fields to the specimen of between 0 and $1.5 \mathrm{~T}$. The electrostatic and magnetic contributions to the phase shift were separated by turning the specimen over inside the electron microscope using a modified Fischione 2050 tomography specimen holder. Off-axis electron holograms were reconstructed numerically using a standard Fourier-transform-based method with sideband filtering using HoloWorks software in the Gatan microscopy suite, as well as using home-written scripts in the Semper image processing language ${ }^{41}$. Contour lines and colour maps 
were generated from recorded magnetic phase images to yield magnetic induction maps.

\section{References}

1. Kramer, M. J., McCallum, R. W., Anderson, I. A., Constantinides, S., Prospects for non-rare earth permanent magnets for traction motors and generators. JOM 64, 752-763 (2012).

2. Zhou, L., Miller, M K., Lu, P., Ke, L., Skomski, R., Dillon, H., Xing, Q., Palasyuk, A., McCartney, M. R., Smith, D. J., Constantinides, S., McCallum, R. W., Anderson, I. E., Antropov, V., Kramer, M. J., Architecture and magnetism of alnico. Acta Mater. 74, 224-233 (2014).

3. McCurrie, R. A., The structure and properties of alnico permanent magnet alloys. Handbook of ferromagnetic materials 3, 107-188 (1982).

4. Hao, S. M., Ishida, K., Nishizawa, T., Role of alloying elements in phase decomposition in alnico magnet alloys Metall. Mater. Trans. 16, 179-185 (1985).

5. Yeh, J. W., Chen, S. K., Lin, S. J., Gan, J. Y., Chin, T. S., Shun, T. T., Tsau, C. H., Chang, S. Y., Nanostructured high-entropy alloys with multiple principal elements: novel alloy design concepts and outcomes. Adv. Eng. Mater. 6, 299-303 (2004).

6. George, E. P., Raabe, D., Ritchie, R. O., High-entropy alloys. Nat. Rev. Mater. 4, 515 (2019).

7. Miracle, D. B., Senkov, O. N., A critical review of high entropy alloys and related concepts. Acta Mater. 122, 448-511 (2017).

8. Li, Z., Pradeep, K. G., Deng, Y., Raabe, D., Tasan, C. C., Metastable high-entropy dual-phase alloys overcome the strength-ductility trade-off. Nature 534, 227 (2016). 
9. Santodonato, L. J,, Zhang, Y., Feygenson, M., Parish, C. M., Gao, M. C., Weber, R. JK, Neuefeind, J. C., Tang, Z., Liaw, P. K., Deviation from high-entropy configurations in the atomic distributions of a multi-principal-element alloy. Nat. Commun. 6, 5964 (2015).

10. Zhang, Y., Zuo, T., Cheng, Y., Liaw, P. K., High-entropy alloys with high saturation magnetization, electrical resistivity, and malleability. Sci. Rep. 3, 1455 (2013).

11. Koželj, P., Vrtnik, S., Jelen, A., Jazbec, S., Jagličić, Z., Maiti, S., Feuerbacher, M., Steurer, W., Dolinšek, J., Discovery of a superconducting high-entropy alloy. Phys. Rev. Lett. 113, 107001 (2014).

12. Senkov, O. N., Miller, J. D., Miracle, D. B., Woodward, C., Accelerated exploration of multiprincipal element alloys with solid solution phases. Nat. Commun. 6, 6529 (2015).

13. Lu, W., Liebscher, C. H., Dehm, G., Raabe, D., Li, Z., Bidirectional transformation enables hierarchical nanolaminate dual-phase high-entropy alloys. Adv. Mater. 30, 1804727 (2018).

14. Miracle, D. B., High entropy alloys as a bold step forward in alloy development. Nat. Commun. 10, 1805 (2019).

15. Jung, C., Kang, K., Marshal, A., Pradeep, K. G., Seol, J.-B., Lee, H. M., Choi, P.-P., Effects of phase composition and elemental partitioning on soft magnetic properties of AlFeCoCrMn high entropy alloys. Acta Mater. 171, 31-39 (2019).

16. Fu, Z., MacDonald, B. E., Dupuy, A. D., Wang, X., Monson, T. C., Delaney, R. E., Pearce, C. J., Hu, K., Jiang, Z., Zhou, Y., Schoenung, J. M., Chen, W., Lavernia, E. J., Exceptional combi- 
nation of soft magnetic and mechanical properties in a heterostructured high-entropy composite, Appl. Mater. Today 15, 590-59 (2019).

17. Rao, Z., Dutta, B., Körmann, F., Ponge, D., Li, L., He, J., Stephenson, L., Schäfer, L., Skokov, K., Gutfleisch, O., Raabe, D., Li, Z., Unveiling the mechanism of abnormal magnetic behavior of FeNiCoMnCu high-entropy alloys through a joint experimental-theoretical study, Phys. Rev. Mater. 4, 014402 (2020).

18. Ma, Y., Wang, Q., Jiang, B. B., Li, C. L., Hao, J. M., Li, X. N., Dong, C., Nieh, T. G., Controlled formation of coherent cuboidal nanoprecipitates in body centered cubic high entropy alloys based on $\mathrm{Al}_{2}(\mathrm{NiCoFeCr})_{14}$ compositions. Acta Mater. 147, 213-225 (2018).

19. Li, P., Wang, A., Liu, C. T., A ductile high entropy alloy with attractive magnetic properties. $J$ Alloy. Compd. 694, 55-60 (2017).

20. Borkar, T., Chaudhary, V., Gwalani, B., Choudhuri, D., Mikler, C. V., Soni, V., Alam, T., Ramanujan. R. V., Banerjee, R., Combinatorial Approach for Assessing the Magnetic Properties of High Entropy Alloys: Role of $\mathrm{Cr}$ in $\mathrm{AlCo}_{x} \mathrm{Cr}_{1-x} \mathrm{FeNi}$. Adv. Eng. Mater. 19, 8, 1700048 (2017).

21. Chaudhary, V., Gwalani, B., Soni, V., Ramanujan, R. V., Banerjee, R., Influence of Cr Substitution and Temperature on Hierarchical Phase Decomposition in the AlCoFeNi High Entropy Alloy. Sci. Rep. 8, 15578 (2018). 
22. Vrtnik, S., Guo, S., Sheikh, S., Jelen, A., Koželj, P., Luzar, J., Kocjan, A., Jagličić, Z., Meden, A., Guim, H., Magnetism of CoCrFeNiZr eutectic high-entropy alloys. Intermetallics 93, 122133 (2018).

23. Cieslak, J., Tobola, J., Reissner, M., The effect of bcc/fcc phase preference on magnetic properties of $\mathrm{Al}_{x} \mathrm{CrFeCoNi}$ high entropy alloys, Intermetallics 118, 106672 (2020).

24. Lucas, M. S., Mauger, L., Muñoz, J. A., Xiao, Y., Sheets, A. O., Semiatin, S. L., Horwath, J., Turgut, Z., Magnetic and vibrational properties of high-entropy alloys. J Appl. Phys. 109, 07E307 (2011).

25. Niu, C., LaRosa, C. R., Miao, J., Mills, M. J., Ghazisaeidi, M., Magnetically-driven phase transformation strengthening in high entropy alloys. Nat. Commun. 9, 1363 (2018).

26. Gureyev, T. E., Roberts, A., Nugent, K. A., Partially coherent fields, the transport-of-intensity equation, and phase uniqueness. J Opt. Soc. Am. A 12, 1942-1946 (1995).

27. Gureyev, T. E., Nugent, K. A., Rapid quantitative phase imaging using the transport of intensity equation. Opt. Commun. 133, 1942-1946 (1995).

28. Dunin-Borkowski, R.E., Kovács, A., Kasama, T., McCartney, M. R., Smith, D. J., Electron holography. Springer Handbook of Microscopy (Eds. Hawkes, P. W., Spence, J. C. H.), Chapter $16,767-806$ (2019).

29. Kovács, A. \& Dunin-Borkowski, R.E., Magnetic imaging of nanostructures using off-axis electron holography. Handbook of magnetic materials (Elsevier, ed. Brück, E.), vol. 27, 59-154 (2018). 
30. Hÿtch, M. J., Dunin-Borkowski, R. E., Scheinfein, M. R., Moulin, J., Duhamel, C., Mazaleyrat, F., Champion, Y., Vortex flux channeling in magnetic nanoparticle chains. Phys. Rev. Lett. 91, 257207 (2003).

31. Kim, M.-K., Dhak, P., Lee, H.-Y., Lee, J.-Y., Yoo, M.-W., Lee, J., Jin, K., Chu, A., Nam, K. T., Park, H. S., Aizawa, S., Tanigaki, T., Shindo, D., Kim, M., Kim, S.-K., Self-assembled magnetic nanospheres with three-dimensional magnetic vortex. Appl. Phys. Lett. 105, 232402 (2014).

32. Caron, J., Model-based reconstruction of magnetisation distributions in nanostructures from electron optical phase images. RWTH Aachen University PhD Thesis, (2017).

33. Shinjo, T., Okuno, T., Hassdorf, R., Shigeto, K., Ono, T., Magnetic vortex core observation in circular dots of permalloy. Science 2895481 930-932 (2000).

34. Midgley, P. A., Dunin-Borkowski, R. E., Electron tomography and holography in materials science. Nat. Mater. 4, 271 (2009).

35. Krishnan, K.M., Fundamentals and applications of magnetic materials, Oxford University Press 9 388-404 (2016).

36. Schneeweiss, O., Friák, M., Dudová, M., Holec, D., Šob, M., Kriegner, D., Holỳ, V., Beran, P., George, E. P., Neugebauer, J., Magnetic properties of the CrMnFeCoNi high-entropy alloy. Phys. Rev. B 96, 014437 (2017).

37. Herzer, G., Grain size dependence of coercivity and permeability in nanocrystalline ferromagnets. IEEE Trans. Magn 26, 1397-1402 (1990). 
38. Ernst Ruska-Centre for Microscopy and Spectroscopy with Electrons (ER-C), Forschungszentrum Jülich and RWTH Aachen, FEI Titan G2 80-200. Journal of large-scale research facilities 2, A43 (2016).

39. Ernst Ruska-Centre for Microscopy and Spectroscopy with Electrons (ER-C), Forschungszentrum Jülich and RWTH Aachen, FEI Titan 60-300 HOLO. Journal of large-scale research facilities 2, A44 (2016).

40. Chang, S. L.Y., Dwyer, C., Barthel, J., Boothroyd, C. B., Dunin-Borkowski, R. E., Performance of a direct detection camera for off-axis electron holography. Ultramicroscopy 161, 9097 (2016).

41. Saxton, P. A., Pitt, T. J., Horner, M., Digital image processing: the Semper system. Ultramicroscopy 3, 343 (1979).

Supplementary information Supplementary Information accompanies this paper at www.nature.com/ncomms.

Acknowledgements Technical support from W. Pieper is gratefully acknowledged. This project has received funding from the European Research Council (ERC) under the European Union's Horizon 2020 research and innovation programme under grant agreements 856538 (3D MAGiC) and 823717 (ESTEEM3), as well as from the DARPA TEE program through grant MIPR\# HR0011831554 and the Deutsche Forschungsgemeinschaft (DFG, German Research Foundation)-Project-ID 405553726-TRR 270. This work is also supported by the AME Programmatic Fund of the Agency for Science, Technology and Research, Singapore under Grant No. A1898b0043. 
Author contributions Q.L. and A.K. conceived and conducted the TEM experiments, S.V., R.B. and V.C. processed the alloys and conducted the APT and bulk measurements. Q.L, J.C., H.D. and D.S. analyzed the results. R.B., R.V.R. and R.E.D.B. provided research guidance. All authors contributed to the writing and reviewed the manuscript.

Competing Interests The authors declare that they have no competing financial interests.

Correspondence Correspondence and requests for materials should be addressed to Q.L. (email: q.lan@fzjuelich.de). 
Figure 1 Magnetization $(M)$ vs applied magnetic field $(H)$ measured at a temperature of $300 \mathrm{~K}$ for the $\mathrm{AlCoFeNi}$ and $\mathrm{AICo}(\mathrm{Cr}) \mathrm{FeNi} \mathrm{HEAs}$. The inset shows a magnified view of the central part of the hysteresis loop.

Figure 2 Microstructure and magnetic texture of the AICoFeNi HEA. (a) HAADF STEM image of two grains, with grain 1 aligned to a zone axis. The orientations of the grains are indicated. (b) Combined $\mathrm{Al}+\mathrm{Fe}$ elemental map obtained from grain 1 using STEM EDXS imaging. (c) Atomic-resolution HAADF STEM image and (d) corresponding EDX map. (e) Overfocus $(100 \mu \mathrm{m})$ Fresnel image recorded from grain 2 and (f) corresponding magnetic induction map reconstructed using the transport-of-intensity equation. The color wheel indicates the direction and magnitude of the projected in-plane magnetic induction.

Figure 3 Phase separation in the AICo(Cr)FeNi HEA. (a) HAADF STEM image of the multi-scale hierarchical B2 and A2 microstructure, containing characteristic R1 and $\mathrm{R} 2$ regions. EDXS elemental maps of $\mathrm{Al}, \mathrm{Cr}, \mathrm{Fe}, \mathrm{Co}$ and $\mathrm{Ni}$ recorded from the marked rectangular area are displayed around the main frame. An orange arrow marks an A2 shell around an R2 island. (b) Schematic diagram of the R1 and R2 phase arrangements. In the R1 phase, fine A2 gen-3 and medium A2 gen-2 precipitates form in a B2 matrix. The A2 gen-2 precipitates are covered by an A2 shell. In region R2, B2 precipitates form in an A2 gen-1 matrix covered by an A2 shell. 
Figure 4 Microstructure and chemical composition of the annealed $\mathrm{AICo}(\mathrm{Cr}) \mathrm{FeNi}$

HEA. (a) 3D APT reconstruction of A2 gen-2 and gen-3 precipitates in a B2 matrix in region R1. An Fe-Co-rich shell forms around the A2 gen-2 precipitates. The colors represent Al (red), Ni (green) and Fe (magenta). (b-d) HAADF STEM image and corresponding Fe and Co elemental maps measured using EDXS imaging. Fe and Co enrichment is evident in the interface region of the two phases. (e-g) Atomic-resolution HAADF STEM images of $A 2$ gen-3 in the B2 matrix, the A2 shell around the A2 gen-2 precipitates (marked by a white rectangle in Fig. 4b) and the A2 shell around an A2 gen-1 island (marked by an orange arrow in Fig. 3a), respectively. (h, i) Compositional profiles across the A2 shells around the A2 gen-2 phase and the A2 gen-3 phase, respectively. Fe and Co enrichment is observed in the shells.

Figure 5 Magnetic microstructure of region R1 containing A2 precipitates in a B2

matrix. (a) Mean inner potential and (b) magnetic contribution to the phase shift $\left(\varphi_{M}\right)$ recorded using off-axis $\mathrm{EH}$ with the specimen in a magnetic remanent state. The magnetic phase shift $\left(\varphi_{M}\right)$ in the A2 gen-2 precipitates is either bright or dark, as a result of the presence of a magnetic vortex in each precipitate. The marked A2 gen-2 precipitates (A and B) are further analysed in (d). (c) Large-field-of-view magnetic induction map derived from the magnetic contribution to the phase shift, showing clockwise and counterclockwise magnetic vortex states in the A2 precipitates. The phase contour spacing is $2 \pi / 24$ rad. (d) Projected in-plane magnetization $\left(M_{x y}\right)$ in the A2 precipitates marked in (b) determined from the magnetic contribution to the phase shift using model-based iterative 
reconstruction. An upper limit for the diameter of the magnetic vortex core, which has an out-of-plane magnetic field orientation, is $8 \mathrm{~nm}$. (e) Sections showing the embedding of A2 precipitates in the B2 matrix extracted from a tomographic reconstruction obtained from a tilt series of ADF STEM images.

\section{Figure 6 Magnetic switching of A2 gen-2 precipitates in the R1 phase in the AICo(Cr)FeNi}

HEA. (a, b) Magnetic contribution to the phase shift $\varphi_{m}$ recorded after returning to remanence from opposite out-of-plane fields of -500 and $500 \mathrm{mT}$, respectively. The localized regions of dark and bright contrast correspond to clockwise or counterclockwise magnetization rotation directions in individual A2 precipitates. Contrast reversal of the A2 gen-2 precipitates is associated with a change in the magnetic field rotation direction of the vortices. In (b), marked precipitates (white squares) retained their magnetization rotation direction from that observed in (a). (c) Magnitude and sign of the local change in magnetic phase shift $\left(\varphi_{M}\right)$ of individual A2 gen-2 precipitates of different size plotted as a function of out-of-plane magnetic field. Positive values are associated with counterclockwise magnetization rotation directions. (d) Line profiles of magnetic phase shift extracted from $(a, b)$ between two A2 gen-2 precipitates, as indicated by red and blue arrows. The step in phase $\Delta \varphi$ indicates a projected in-plane magnetic field contribution from the B2 matrix.

Figure 7 Magnetic microstructure of a B2 + A2 solid solution mixture in regions $\mathrm{R} 1$ and $\mathrm{R} 2$ and the $\mathrm{A} 2$ shell of the of $\mathrm{AlCo}(\mathrm{Cr}) \mathrm{FeNi} H E A$. (a) Fresnel defocus image 
recorded at remanence. The defocus value used was $-200 \mu \mathrm{m}$. (b) Mean inner potential contribution to the phase measured using off-axis $\mathrm{EH}$ from the $\mathrm{R} 2(+\mathrm{A} 2$ shell) region between two R1 regions. (c) The corresponding magnetic induction map. The phase contour spacing is $2 \pi / 16$ rad. Selected B2 inclusions in the A2 gen-1 matrix are marked with white frames in (b) and (c). The colors and arrows mark the projected in-plane magnetic field direction. (d) Line profile of the magnetic phase shift across an $\mathrm{R} 2$ region that includes A2 shell regions and a single B2 precipitate. The dip in the middle of the phase shift profile is associated with a weakly magnetic or non-magnetic B2 phase. (e) Line profile of the magnetic phase shift, in which the slope in an $A 2$ shell region $(0.075 \mathrm{rad} / \mathrm{nm})$ is $25 \%$ higher than that in an $\mathrm{R} 2$ region $(0.06 \mathrm{rad} / \mathrm{nm})$. 
Figures

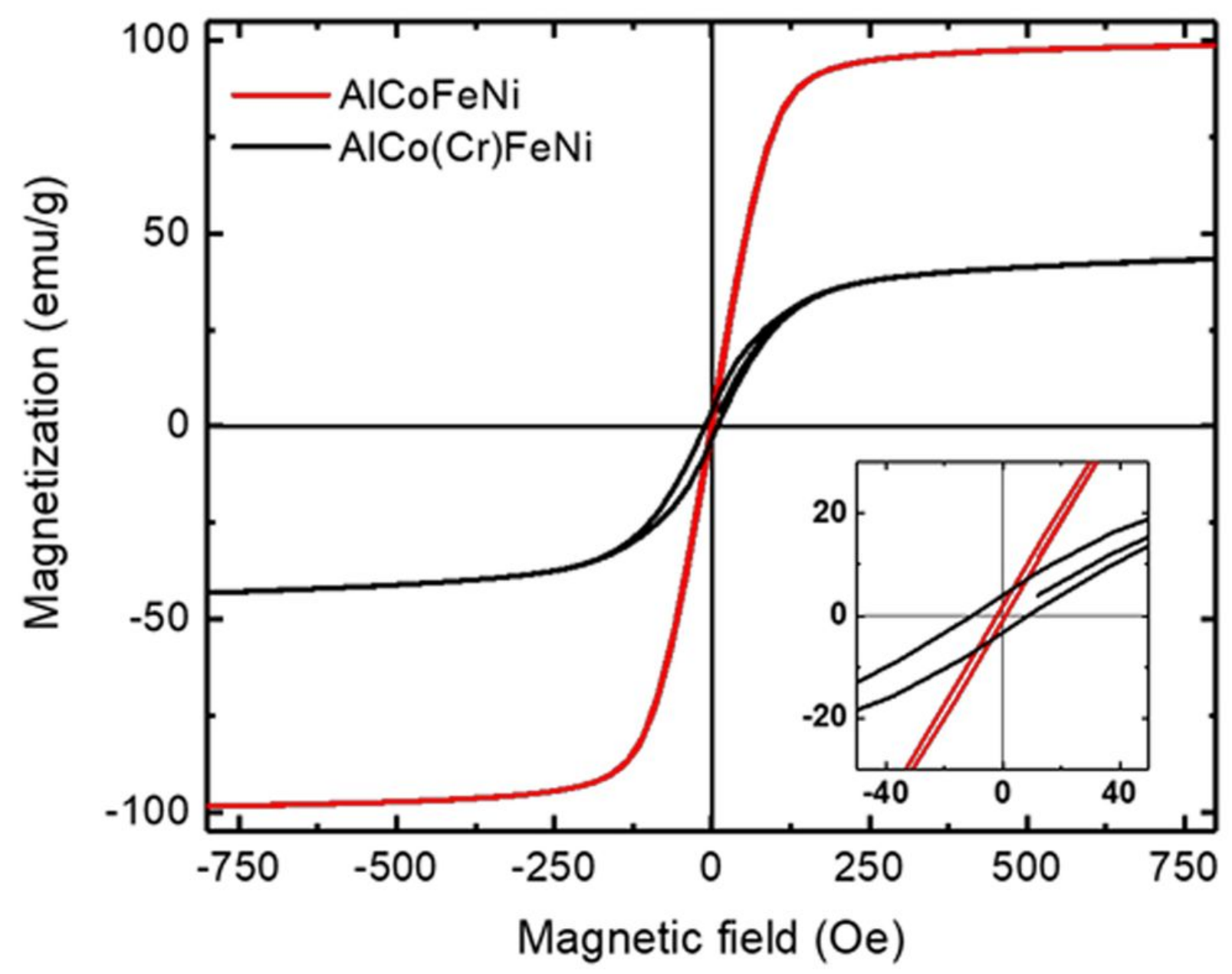

Figure 1

Magnetization (M) vs applied magnetic field $(\mathrm{H})$ measured at a temperature of $300 \mathrm{~K}$ for the AlCoFeNi and $\mathrm{AlCo}(\mathrm{Cr}) \mathrm{FeNi} \mathrm{HEAs}$. The inset shows a magnified view of the central part of the hysteresis loop. 

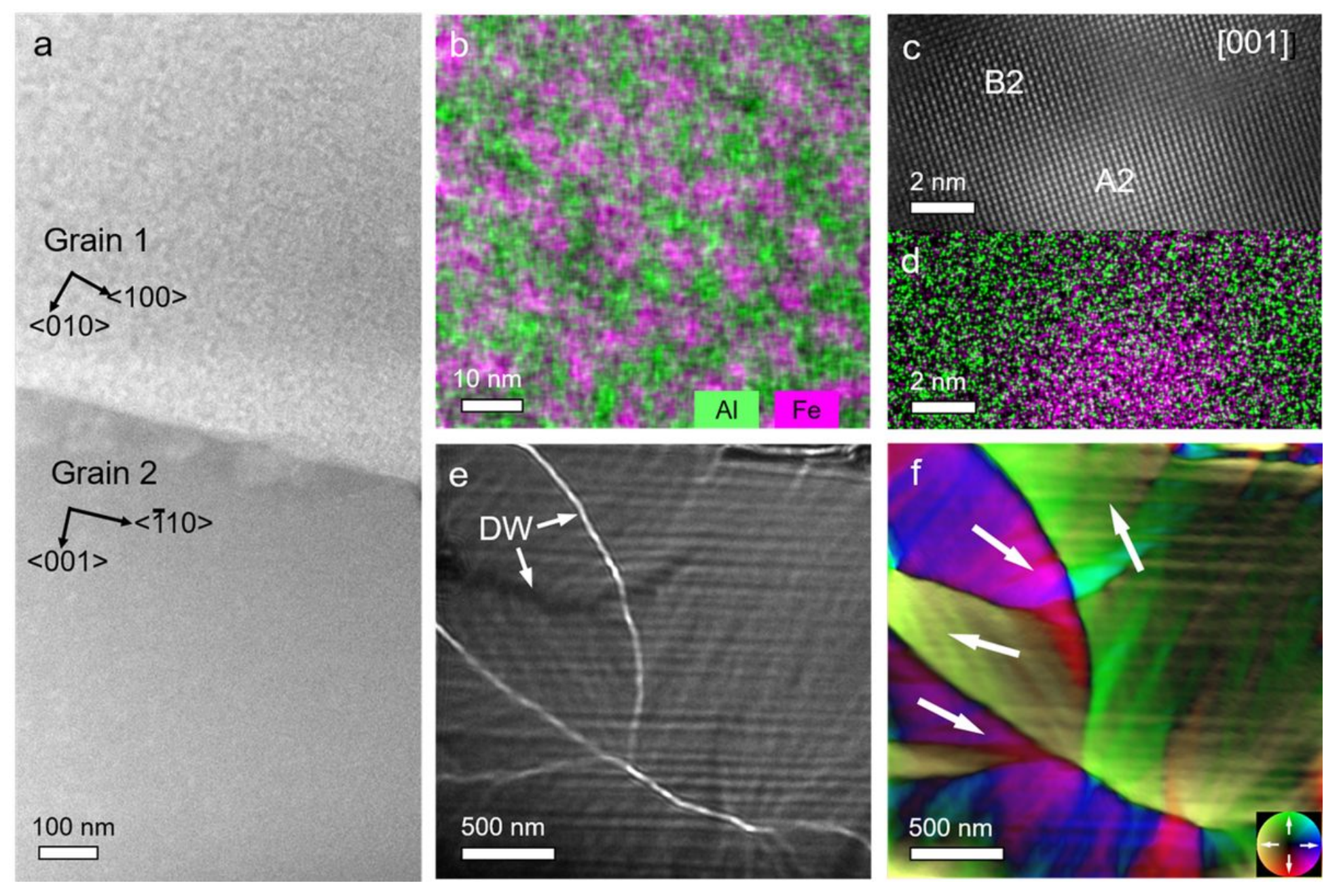

Figure 2

Microstructure and magnetic texture of the AICoFeNi HEA. (a) HAADF STEM image of two grains, with grain 1 aligned to a zone axis. The orientations of the grains are indicated. (b) Combined Al+Fe elemental map obtained from grain 1 using STEM EDXS imaging. (c) Atomic-resolution HAADF STEM image and (d) corresponding EDX map. (e) Overfocus ( $100 \rrbracket \mathrm{m})$ Fresnel image recorded from grain 2 and (f) corresponding magnetic induction map reconstructed using the transport-of-intensity equation. The color wheel indicates the direction and magnitude of the projected in-plane magnetic induction. 

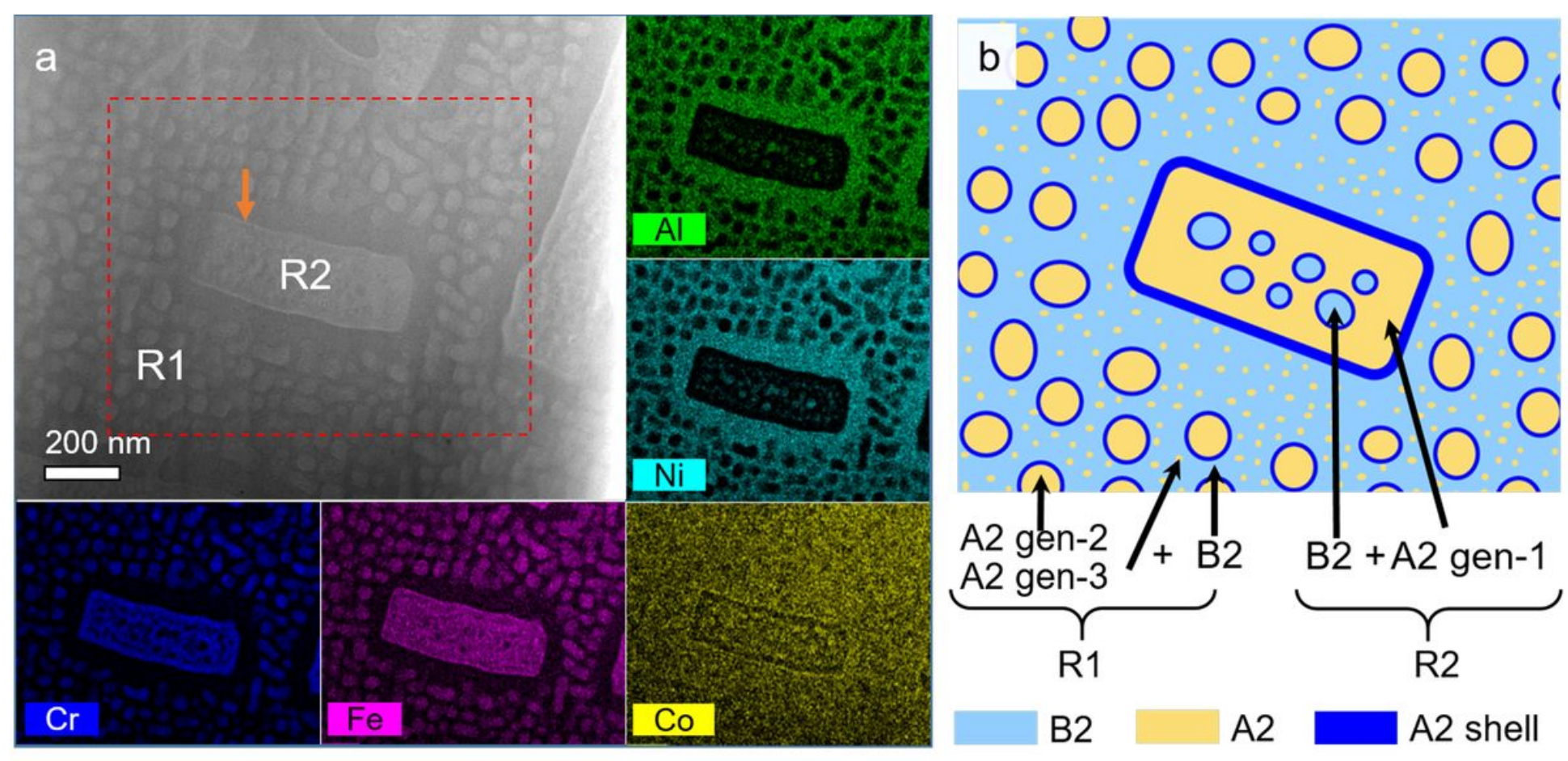

Figure 3

Phase separation in the AlCo(Cr)FeNi HEA. (a) HAADF STEM image of the multi-scale hierarchical B2 and A2 microstructure, containing characteristic R1 and R2 regions. EDXS elemental maps of $\mathrm{Al}, \mathrm{Cr}, \mathrm{Fe}, \mathrm{Co}$ and $\mathrm{Ni}$ recorded from the marked rectangular area are displayed around the main frame. An orange arrow marks an A2 shell around an R2 island. (b) Schematic diagram of the R1 and R2 phase arrangements. In the R1 phase, fine A2 gen-3 and medium A2 gen-2 precipitates form in a B2 matrix. The A2 gen-2 precipitates are covered by an A2 shell. In region R2, B2 precipitates form in an A2 gen-1 matrix covered by an A2 shell. 

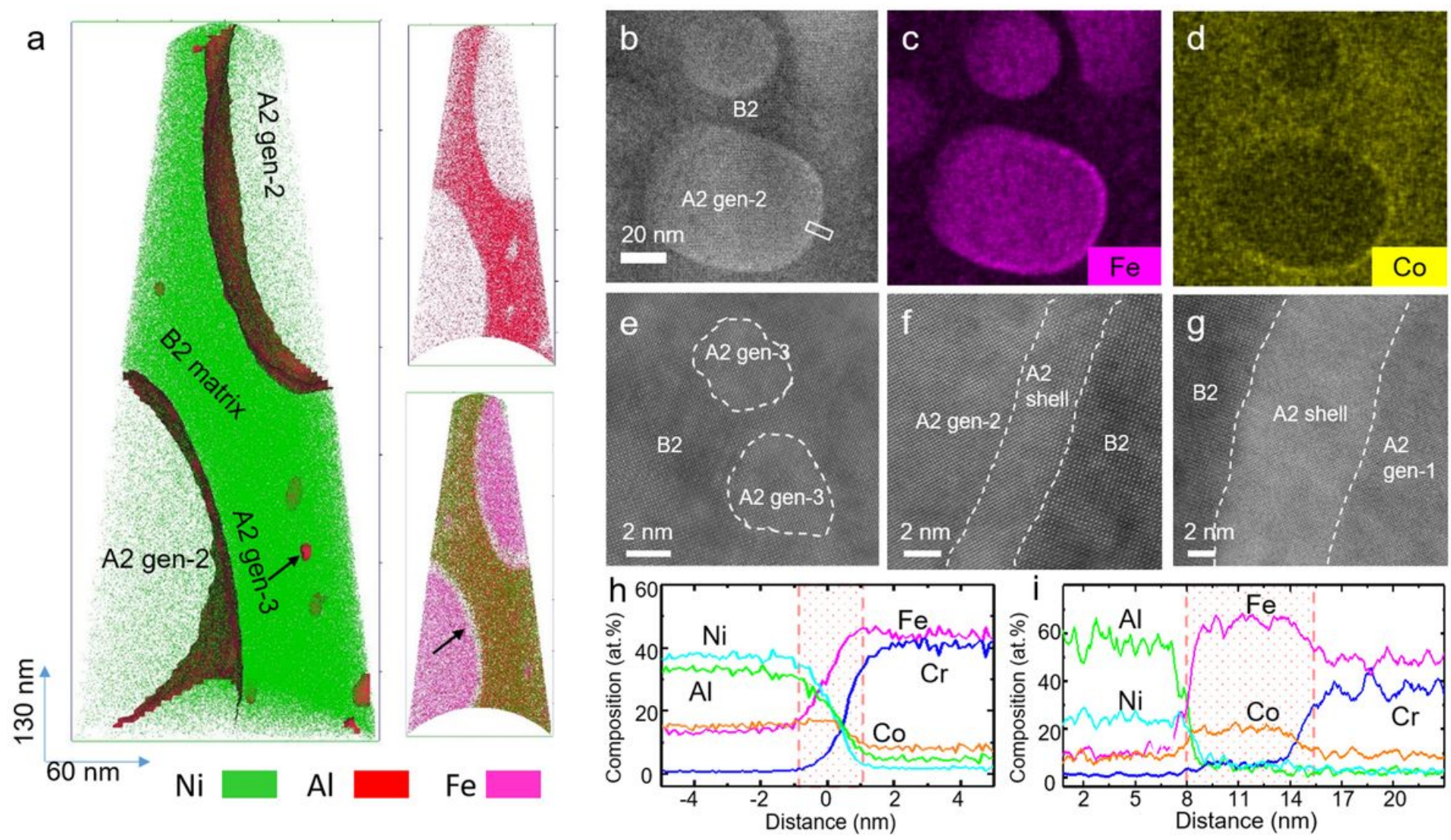

Figure 4

Microstructure and chemical composition of the annealed AlCo(Cr)FeNi HEA. (a) 3D APT reconstruction of A2 gen-2 and gen-3 precipitates in a B2 matrix in region R1. An Fe-Co-rich shell forms around the A2 gen-2 precipitates. The colors represent Al (red), Ni (green) and Fe (magenta). (b-d) HAADF STEM image and corresponding Fe and Co elemental maps measured using EDXS imaging. Fe and Co enrichment is evident in the interface region of the two phases. (e-g) Atomic-resolution HAADF STEM images of A2 gen3 in the B2 matrix, the A2 shell around the A2 gen-2 precipitates (marked by a white rectangle in Fig. 4b) and the A2 shell around an A2 gen-1 island (marked by an orange arrow in Fig. 3a), respectively. $(\mathrm{h}, \mathrm{i})$ Compositional profiles across the A2 shells around the A2 gen-2 phase and the A2 gen-3 phase, respectively. Fe and Co enrichment is observed in the shells. 

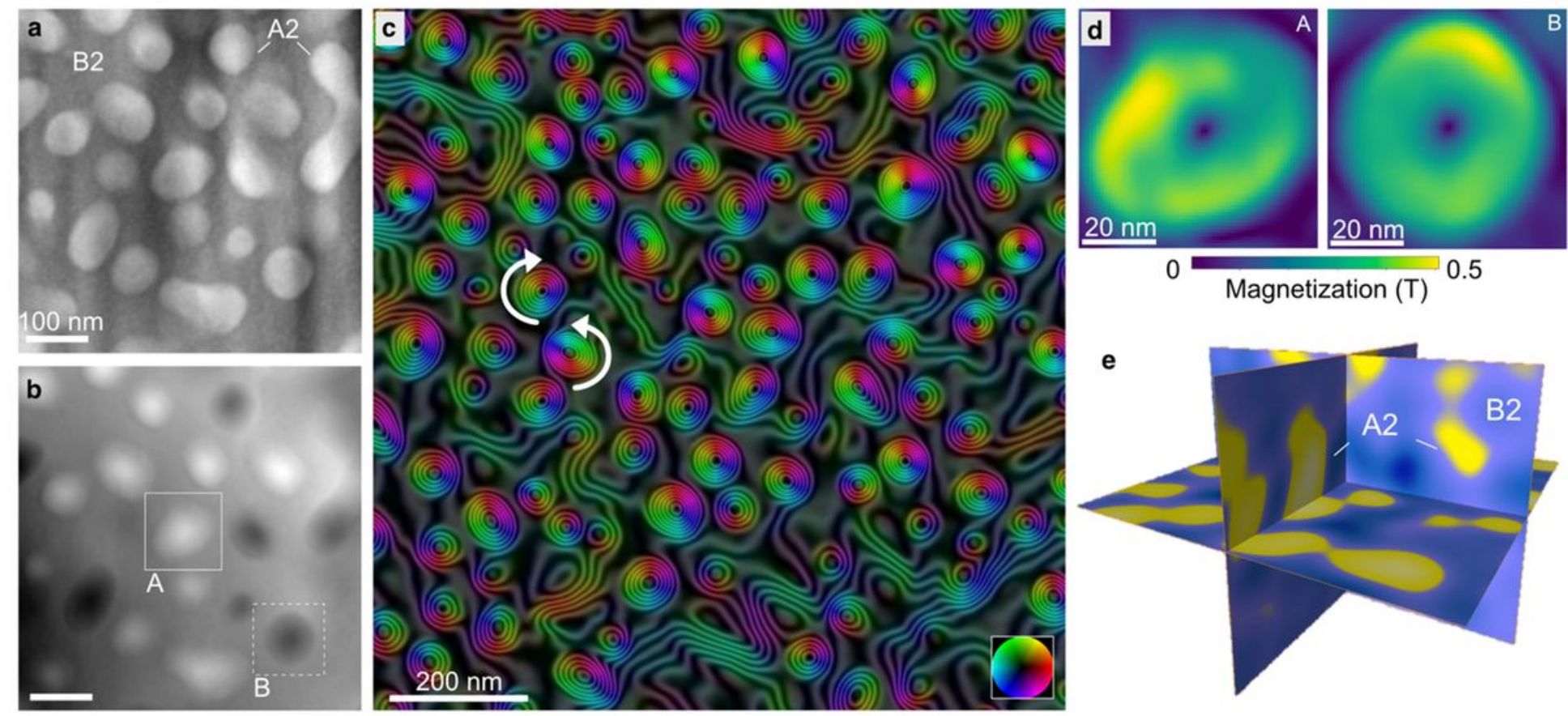

Figure 5

Magnetic microstructure of region R1 containing A2 precipitates in a B2 matrix. (a) Mean inner potential and (b) magnetic contribution to the phase shift ('M) recorded using off-axis EH with the specimen in a magnetic remanent state. The magnetic phase shift ('M) in the A2 gen-2 precipitates is either bright or dark, as a result of the presence of a magnetic vortex in each precipitate. The marked A2 gen-2 precipitates (A and B) are further analysed in (d). (c) Large-field-of-view magnetic induction map derived from the magnetic contribution to the phase shift, showing clockwise and counterclockwise magnetic vortex states in the $\mathrm{A} 2$ precipitates. The phase contour spacing is $2 \mathbb{2} / 24$ rad. (d) Projected in-plane magnetization ( $\mathrm{Mxy}$ ) in the $\mathrm{A} 2$ precipitates marked in (b) determined from the magnetic contribution to the phase shift using model-based iterative 27 reconstruction. An upper limit for the diameter of the magnetic vortex core, which has an out-of-plane magnetic field orientation, is $8 \mathrm{~nm}$. (e) Sections showing the embedding of $A 2$ precipitates in the $B 2$ matrix extracted from a tomographic reconstruction obtained from a tilt series of ADF STEM images. 

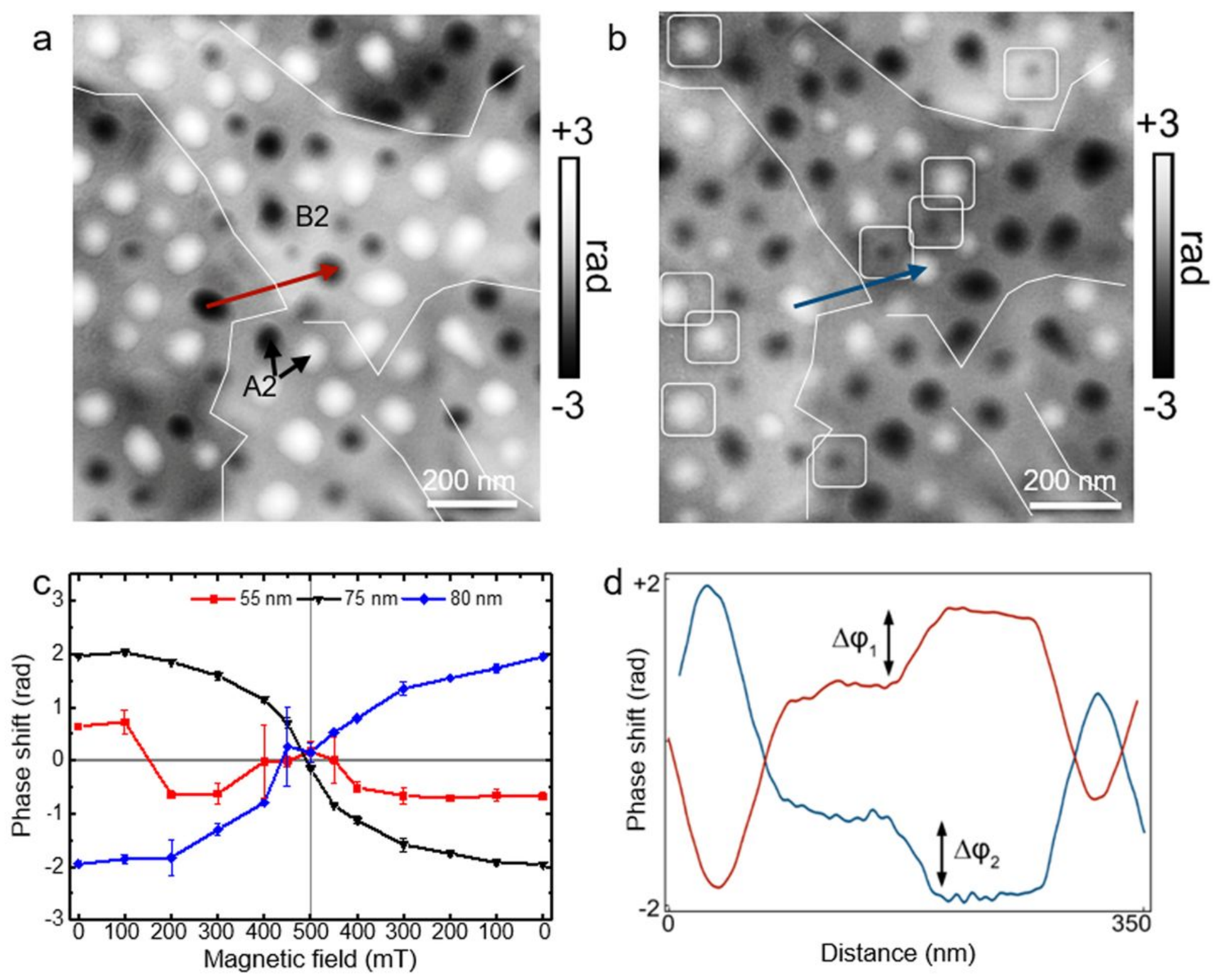

Figure 6

Magnetic switching of A2 gen-2 precipitates in the R1 phase in the AlCo(Cr)FeNi HEA. (a, b) Magnetic contribution to the phase shift ' $m$ recorded after returning to remanence from opposite out-of-plane fields of -500 and $500 \mathrm{mT}$, respectively. The localized regions of dark and bright contrast correspond to clockwise or counterclockwise magnetization rotation directions in individual A2 precipitates. Contrast reversal of the A2 gen-2 precipitates is associated with a change in the magnetic field rotation direction of the vortices. In (b), marked precipitates (white squares) retained their magnetization rotation direction from that observed in (a). (c) Magnitude and sign of the local change in magnetic phase shift ('M) of individual A2 gen-2 precipitates of different size plotted as a function of out-of-plane magnetic field. Positive values are associated with counterclockwise magnetization rotation directions. (d) Line profiles of magnetic phase shift extracted from $(a, b)$ between two A2 gen-2 precipitates, as indicated by red and blue arrows. The step in phase $\mathbb{Q}^{\prime}$ indicates a projected in-plane magnetic field contribution from the B2 matrix. 

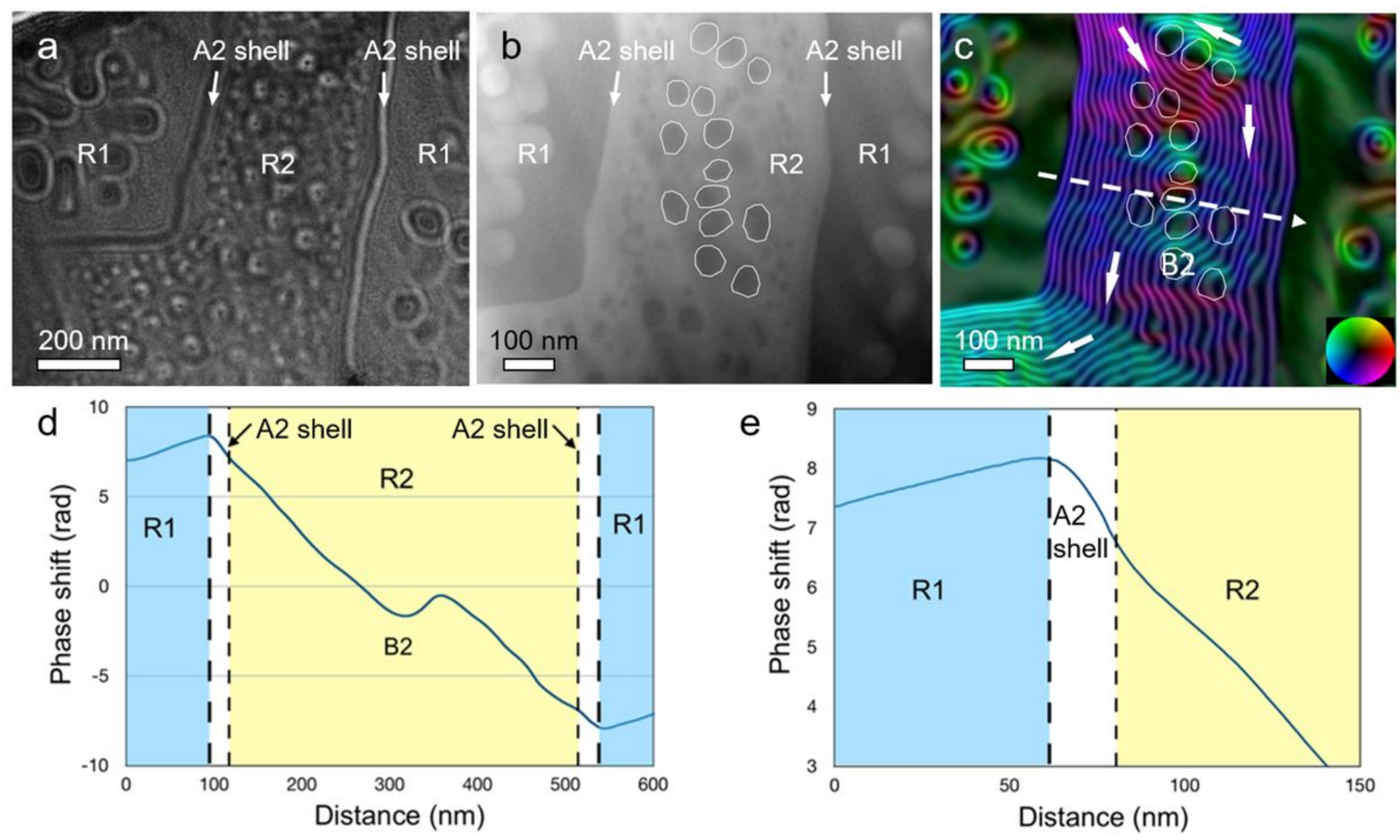

\section{Figure 7}

Magnetic microstructure of a B2 + A2 solid solution mixture in regions R1 and R2 and the A2 shell of the of AlCo(Cr)FeNi HEA. (a) Fresnel defocus image 28 recorded at remanence. The defocus value used was $-200 \llbracket m$. (b) Mean inner potential contribution to the phase measured using off-axis EH from the R2 (+A2 shell) region between two R1 regions. (c) The corresponding magnetic induction map. The phase contour spacing is $2 \nabla / 16$ rad. Selected B2 inclusions in the A2 gen-1 matrix are marked with white frames in (b) and (c). The colors and arrows mark the projected in-plane magnetic field direction. (d) Line profile of the magnetic phase shift across an $\mathrm{R} 2$ region that includes A2 shell regions and a single B2 precipitate. The dip in the middle of the phase shift profile is associated with a weakly magnetic or non-magnetic B2 phase. (e) Line profile of the magnetic phase shift, in which the slope in an A2 shell region (0.075 $\mathrm{rad} / \mathrm{nm})$ is $25 \%$ higher than that in an R2 region $(0.06 \mathrm{rad} / \mathrm{nm})$.

\section{Supplementary Files}

This is a list of supplementary files associated with this preprint. Click to download.

- Supplementaryinformation.pdf 